\title{
Microstructure-Aided Digital Volume Correlation
}

\author{
H. Leclerc ${ }^{1, a}$, J.-N. Périé2 ${ }^{2}$ A. Fanget ${ }^{3}$, E. Maire ${ }^{4}$, F. Hild ${ }^{1}$, and S. Roux ${ }^{1}$
}

1 LMT-Cachan, ENS Cachan / CNRS / UPMC / PRES UniverSud, 61 avenue du Président Wilson, F-94235 Cachan Cedex, France

2 ICA (Institut Clément Ader); Université de Toulouse, INSA, UPS, Mines Albi, ISAE, 133 avenue de Rangueil, F-31077 Toulouse, France

3 Centre d'Etudes de Gramat (CEA-DAM), F-46500 Gramat, France

4 Laboratoire Matériaux, Ingénierie et Sciences (MATEIS), INSA-Lyon / CNRS

7 avenue Jean Capelle, F-69621 Villeurbanne, France

\begin{abstract}
This paper describes how volumic images of a composite material could be used to measure 3D displacement fields, and assess mechanical properties. The exemple of a tensile test performed on a sample made of an energetic material is chosen. Different tools are used, namely, X-ray microtomography of an in situ experiment, image acquisition and processing, volume correlation to measure 3D displacement fields. The principle of an integrated approach is finally introduced.
\end{abstract}

\section{Introduction}

Many biological tissues (e.g., bones) and composite materials (e.g., 3D woven composites) exhibit complex, coarse and nonperiodic microstructures. For such heterogeneous materials, classical homogenization techniques may not be applicable. The quest for microstructure dependent macroscopic behaviour and its identification are then a challenge [1]. The assessment of accurate local strains or stresses from a given macroscopic loading and some microstructural properties may also reveal to be limiting.

The use of emerging 3D imaging techniques such as computed microtomography (CMT), offering a unique access to microstructural features, opens the way to new multiscale approaches. The example of an in situ tensile test performed on an energetic material is used. On the one hand, Finite Element

\footnotetext{
a e-mail: hugo.leclerc@lmt.ens-cachan.fr
} 
(FE) models can be constructed directly from 3D images [2]. On the other hand, Digital Volume Correlation may be used to measure 3D displacement fields in the bulk [3]. The analysis of mechanical tests on energetic materials was already performed by using 2D Digital Image Correlation (DIC). The natural texture may be exploited to get macroscopic displacement fields [4], e.g., to evaluate the minimum size of a representative volume element [5]. A random pattern can also been sprayed onto the surface to determine in-plane displacements at the grain scale, e.g., to analyze cracks [6]. To the authors' best knowledge, 3D analyses of such materials were never performed before.

Section 2 presents the material and the experimental setup. In Section 3, the basic principle of digital image based FE models is introduced. Some meshes automatically generated from the reference scan acquired are presented. In Section 4, a Galerkin approach to Digital Volume Correlation (DVC) [7] is presented. It is used to measure 3D displacements and strain fields during a tensile test. The last section introduces the principal of an integrated DVC approach, coupling global DVC and Finite Element Model Updating. The proposed technique aims at regularizing measured displacement fields and identifying mechanical properties.

\section{Material and experimental setup}

Figure 1 shows a reference volume of the studied pressed energetic material. It is a two-phase composite containing large stiff and brittle grains (i.e., the aggregates) embedded in a compliant viscoelastic binder (i.e., the matrix). The tensile tests were performed in situ at the European Synchrotron Radiation Facility, Grenoble, France (on beamline ID19). X-Ray microtomography enables 3D pictures of the local density of solids to be obtained by exploiting the attenuation of a monochromatic X-ray beam. The dynamic range of the reconstructed volumes (or scans) is equal to 8 bits, and the physical size of one voxel is $7.4 \mu \mathrm{m}$. Six scans were acquired for the analyzed test. Apart from the reference scan, i.e., before any load was applied, three scans were taken up to the peak load and one thereafter (see figure 2). The last one is obtained after failure and is not analyzed herein.

Image and/or volume correlations require a random texture to measure displacements. The grains are extremely helpful for the process, as they constitute a random marking with a high contrast distributed within the specimen. As can be seen in Figure 1, they appear as white clusters with a well defined morphology. The mean gray level is 157 and the corresponding standard deviation is 56, which is a high value due to the bimodal distribution. The size of the reconstructed volumes is $2000^{3}$ voxels, and the analyzed volume of interest (VOI) is centered and has a restricted size because of memory limitations (all the results presented herein are performed on a standard PC). 


\section{Digital image based micro FE modeling}

Many Digital Image Based (DIB) approaches have been proposed [8,9,2,10], especially in the field of biomechanics. For the two-phase material studied in this work and shown in Figure 1, one can for example resort to image segmentation in order to separate the matrix and the inclusions. An FE model can then be automatically generated [11]. The mesh can either be regular (based on voxels) or unstructured (and based on tetrahedral elements). An automated procedure is used to mesh both constituents [12] using tetrahedral elements (Figure 1). In such cases, the behaviour of an element only depends on the local phase. However, the actual state and behaviour of the constituents can generally hardly be assessed (e.g., because of a complex process) or are totally unknown (e.g., in biological tissues).
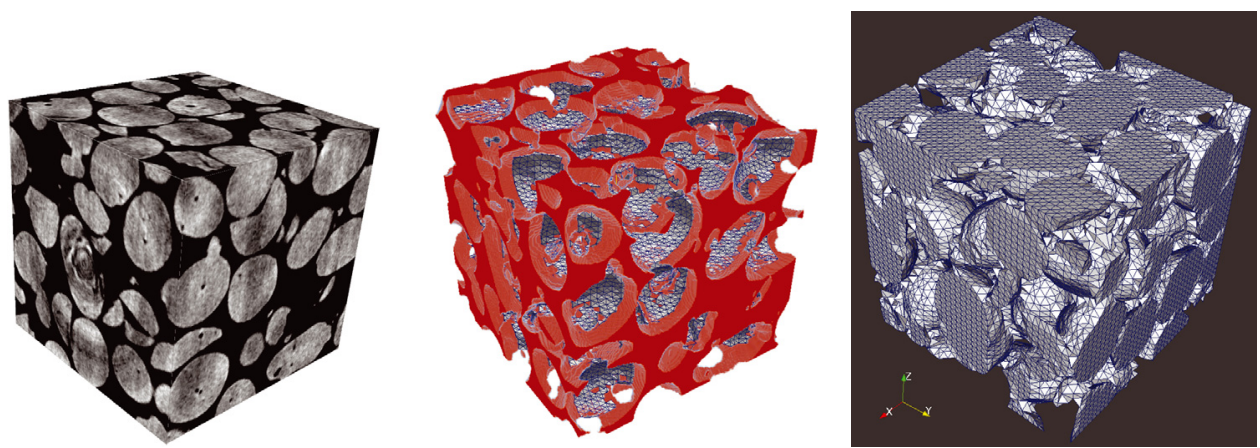

Fig. 1. Study of a polymer bonded particulate composite by using digital image based modeling. From left to right: 1) Raw reference CMT image of the VOI; 2) Result of the segmentation and mesh associated with the matrix; 3) mesh of the inclusions.

The identification of the microconstituent in situ mechanical properties has to be performed directly from a macroscopic test performed on a composite specimen. Mean strains and forces may be measured, and, finally, an inverse problem is solved. The (micro)mechanical parameters are then unknown while the microstructure and experimental (macro)quantities are known. This procedure is fragile. Identification may be influenced by non representative boundary conditions or a poor DIB reconstruction. An alternative route based on DVC measurements and suited to coarse microstructures is proposed in Section 5. 


\section{Finite Element Digital Volume Correlation}

Volume correlation consists in registering the texture of two pictures (or scans in the present case) with the help of a displacement field to be determined. To estimate the unknown displacement field $\mathbf{U}$, the quadratic difference $\varphi^{2}(\mathbf{X})=[f(\mathbf{X})-g(\mathbf{X}+\mathbf{U}(\mathbf{X}))]^{2}$ is integrated over the studied VOI $\Omega$

$$
\Phi^{2}=\int_{\Omega} \varphi^{2}(\mathbf{X}) d \mathbf{X}
$$

and minimized with respect to the degrees of freedom of the measured displacement field [7]. In the present case, a 3D finite element kinematics is chosen [13] for the sought fields. The proposed technique is therefore a Galerkin approach to DVC. It allows for the decomposition of the displacement field onto any meaningful or convenient basis, e.g., a finite element basis (thus fully compatible with the simulations and with DIB procedure). Other approaches to DVC can be found. Namely, as in 2D applications, the most commonly used correlation algorithms consist in registering locally small subvolumes in a sequence of pictures to determine local displacement components [15]. The same type of hypotheses are made in 3D algorithms [3,16-18]. However, the present procedure can naturally be coupled with FE simulation tools.

When dealing with structured meshes, simple shape functions may be used, namely, trilinear polynomials associated with 8-node cube elements (or C8-DIC $[7,14]$ ). When studying complex geometries or microstructures, unstructured meshes based on tetrahedral elements are more relevant. An example of such mesh and the corresponding displacement field measured at the first step of loading are presented in Figure 2. In that case, decreasing the element size first induces a better local description of the displacement fields (checked by using residual maps $\varphi(\mathbf{X})$ ). However, very small elements (typically smaller than grains) cannot be used since no relevant texture will be available, and the measurement uncertainty would not allow for any reliable result to be found.

From the measured displacement fields, the strain fields are computed. In the present case, the infinitesimal strain assumption cannot be made locally. Consequently, a large transformation framework is used. Mean strains over the whole cubic VOI are subsequently determined. Figure 2 presents the change of averaged principal strains with respect to the applied load. An apparent Poisson's ratio $v$ can be estimated in the chosen gauge volume. For the first three load steps, the corresponding Poisson's ratios are $0.50,0.49,0.40$. Up to the peak load, the value of the latter is virtually identical and corresponds to that of an incompressible material. Beyond the peak load, it decreases, signaling displacement heterogeneities. In particular, the volumetric strain $\bar{\epsilon}_{v}=\bar{\epsilon}_{1}+\bar{\epsilon}_{2}+\bar{\epsilon}_{3}$ remains vanishingly small except for the last analyzed level, where it becomes significantly positive (i.e., dilatancy is observed). 

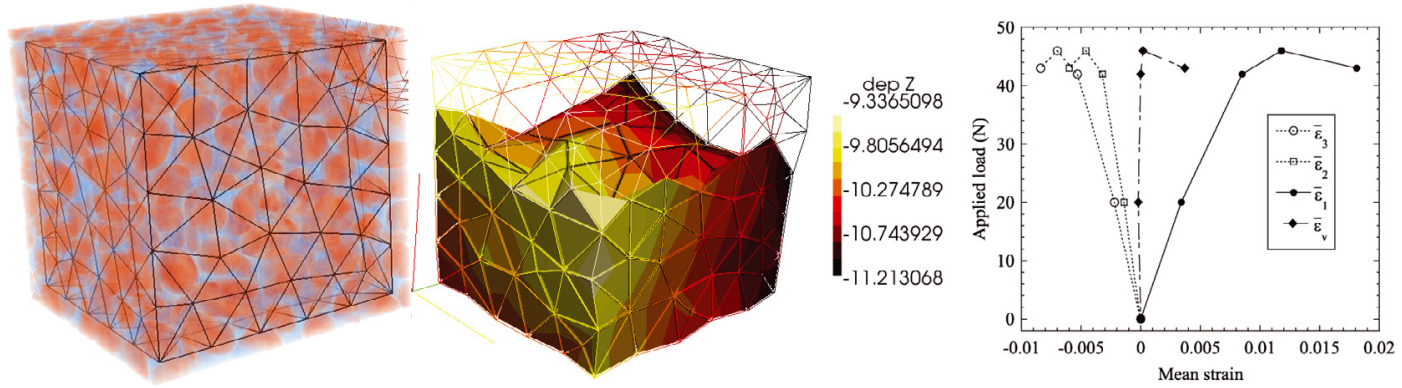

Fig. 2. Study of a polymer bonded particulate composite: FE-DVC using an unstructured mesh. From left to right: 1) unstructured mesh used for DVC; 2) deformed mesh and corresponding displacement contours in the tensile direction; 3 ) change of mean principal $\epsilon_{i}$ and volumetric strains $\epsilon_{v}$ (over the meshed VOI) with the applied macroscopic tensile load.

\section{Conclusion and perspectives}

As shown above, FE-DVC is a useful tool for investigating the multiscale behavior of materials exhibiting complex and coarse architectures. The technique also offers a great opportunity to compare directly computed and measured displacement fields, i.e., to retrieve macromechanical properties from full-field measurements [19]. A common identification technique consists in updating some parameters of the FE model by comparing measured and computed kinematic fields (Finite Element Model Updating or FEMU [20]). A convenient technique to deal with displacement fields consists in applying measured displacement fields on the external surfaces of the volume of interest (VOI) as Dirichlet boundary conditions in the FE simulation. The displacement field inside the VOI is then used to tune the constitutive parameters.

Unfortunately, especially in this case, the texture or contrasts inside the micro constituents are not significant enough to assess accurately the micro kinematic field. One has also to remember that the measured (by FE-DVC) and the computed displacement fields (DIB micro FE model) may not be comparable at the constituent scale. An alternative route has recently been proposed [21]. It consists in using an integrated digital image correlation technique. The technique was first applied with 2D pictures to identify a Poisson's ratio from a biaxial test. The idea is to exploit computed displacement fields, which are meaningful from a mechanical standpoint, to register the reference image onto the deformed one. In practice, the method couples strongly FE-DVC with FEMU.

The approach is presently being tested on the presented energetic material. The displacement field basis used in the global DVC approach then results from a 3D simulation using the constructed (i.e., DIB-based) FE model. The boundary conditions applied on the faces of the FE model are then given 
by FE-DVC measurements achieved by using a coarser mesh (the element size here is typically of the order of the inclusion size, see Figure 2). This work is in progress.

\section{References}

1. B. Banerjee and D. O. Adams, Physica B: Condensed Matter 338, (2003) 8-15.

2. S.J. Holister and N. Kikuchi, Biotechnology and Bioengineering 43,(1994) 11

3. B.K. Bay, T.S. Smith, D.P. Fyhrie, M. Saad, Experimental Mechanics 39, (1999) 10

4. P. J. Rae, S. J. P. Palmer, H. T. Goldrein, A. L. Lewis and J. E. Field, Optics and Lasers in Engineering 41, (2004) 635-648.

5. C. Liu, Experimental Mechanics 45 [3], (2005) 238-243.

6. M. Li, J. Zhang, C. Xiong, J. Fang, J. M. Li, and Y. Hao, Optics and Lasers in Engineering 43 [8], (2005) 856-868.

7. S. Roux, F. Hild, P. Viot, D. Bernard, Composites Part A 39, (2008) 495-508

8. S. C. Cowin, Mechanics of Materials 4 [2], (1985) 137-147.

9. J. Keyak, J. Meagher, H. Skinner, and C.D. Mote J., Journal of Biomedical Engineering 12 [5], (1990) 389-397.

10. van Rietbergen, B., Weinans, H., Huiskes, R. and Odgaard, A., Journal of Biomechanics 28, (1995) 69-81.

11. P. Young, T. Beresford-West, S. Coward, B. Notarberardino, B. Walker, and A. Abdul-Aziz, Philosophical Transactions of the Royal Society A: Mathematical, Physical and Engineering Sciences 366 [1878], (2008) 3155-3173.

12. H. J. Kim and C. C. Swan, International Journal for Numerical Methods in Engineering 58 [11], (2003) 1683-1711.

13. O. C. Zienkievicz and R. L. Taylor, The Finite Element Method, (McGraw-Hill, London (UK), 4th edition, 1989).

14. A. Benoit, S. Guérard, B. Gillet, G. Guillot, F. Hild, D. Mitton, J. Périé, and S. Roux, Journal of Biomechanics 42 [14], (2009) 2381-2386.

15. P. Cheng, M. A. Sutton, H. W. Schreier and S. R. McNeill, Experimental Mechanics 42 [3], (2002) 344-352.

16. T. S. Smith, B. K. Bay and M. M. Rashid, Experimental Mechanics 42 [3], (2002) 272-278.

17. M. Bornert, J.-M. Chaix, P. Doumalin, J.-C. Dupré, T. Fournel, D. Jeulin, E. Maire, M. Moreaud and H. Moulinec, Inst. Mes. Métrol. 4, (2004) 43-88. 
18. E. Verhulp, B. van Rietbergen and R. Huiskes, Journal of Biomechanics 37 [9], (2004) 1313-1320.

19. S. Avril, M. Bonnet, A. Bretelle, M. Grédiac, F. Hild, P. Ienny, F. Latourte, D. Lemosse, S. Pagano, E. Pagnacco, and F. Pierron, Experimental Mechanics 48 [4], (2008) 381-402.

20. K. T. Kavanagh and R. W. Clough, International Journal of Solids and Structures 7, (1971) 13

21. H. Leclerc, J.-N. Périé, S. Roux, F. Hild, Proceedings of the 4th International Conference on Computer Vision/Computer Graphics Collaboration Techniques, Lecture Notes In Computer Science 5496, (2009) 11 